\title{
Multidetector Computed Tomography Evaluation of Uncommon Acute Post-Caesarean Section Complications
}

\author{
Musaib Ahmad Dar ${ }^{1}$, Suhail Rafiq ${ }^{1}$, Sheema Posh ${ }^{2}$, Imran Wagay ${ }^{1}$
}

\begin{abstract}
Introduction. The most common major abdominal surgery in women is caesarean section. Despite being a safe procedure, a variety of complications, both acute and chronic, can occur. About $14.5 \%$ of caesarean sections result in complications. Infection followed by postpartum haemorrhage is the most frequent complication. Imaging modalities such as ultrasonography and multidetector computed tomography are often used in the evaluation of suspected uncommon post-caesarean complications. Computed tomography has been found to be a good initial modality for assessing acute postoperative complications after caesarean delivery.

The objective of the research was to evaluate the imaging findings and the characteristic visual manifestations of atypical acute complications of caesarean section, other than common complications such as postpartum haemorrhage, wound infection, etc.

Materials and Methods. This prospective study was carried out at the Department of Radiodiagnosis and Imaging, Government Medical College, Srinagar from June 2019 to February 2020 in collaboration with the Department of General Surgery and Gynaecology and Obstetrics of the Sher-i-Kashmir Institute of Medical Sciences. All patients with suspected complication in the immediate post-caesarean period were evaluated with contrast-enhanced multidetector computed tomography.

Results. Out of 427 patients who underwent caesarean section, 25 patients were suspected of having uncommon acute complications. Out of 25 patients evaluated for suspected immediate post-caesarean complication, only 5 patients had bladder flap hematoma, 8 patients were diagnosed with uterine dehiscence, 6 patients had uterine rupture, 3 patients suffered from ureteral injury, 1 patient had gossypiboma, 2 patients developed pelvic sepsis. Conclusions. Multidetector computed tomography plays an important role in detection and confirmation of multiple acute complications after caesarean delivery and can also help in guiding the management of complications as well.
\end{abstract}

\section{Keywords}

Multidetector Computed Tomography (MDCT); Caesarean Section; Abscess; Hematoma; Uterine Rupture; Gossypiboma

${ }^{1}$ Department of Radiodiagnosis and Imaging, Government Medical College, Srinagar, Jammu and Kashmir, India

${ }^{2}$ Department of Obstetrics and Gynaecology, Sher-i-Kashmir Institute of Medical Sciences, Srinagar, Jammu and Kashmir, India

*Corresponding author: sheema.posh@gmail.com

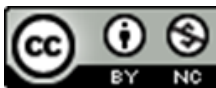

Copyright @Musaib Ahmad Dar, Suhail Rafiq, Sheema Posh, Imran Wagay, 2021

\section{Problem statement and analysis of the latest research}

The most common major abdominal surgery in women is caesarean section. Its highest rate is in Brazil (43.9\%) and the lowest one is in Africa (3\%-5\%) [1-3] with a reported global estimation of $15 \%$ [4]. Various indications for caesarean section include fetal malpresentation and distress, labour dystocia, multiple pregnancy, patient's, or physician's preference [5]. Despite being a safe procedure, a variety of complications, both acute and chronic, can occur. Various acute complications of caesarean section are pelvic hematomas, pelvic abscesses, uterine dehiscence or rupture, pelvic thrombophlebitis. About $14.5 \%$ of caesarean sections result in 
complications [6]. Infection followed by postpartum haemorrhage is the most frequent complication. The increase in the number of complications is due to an increase in the number of emergency caesarean sections, as well as sections performed by residents rather than by experienced senior doctors and lifestyle changes with an increased incidence of obesity which acts as a risk factor that predisposes patients to postoperative complications. Multiple caesarean sections, uterine hypoplasia, history of previous uterine operations such as myomectomy are known to be the risk factors for uterine rupture [7]. Other risk factors include anaemia, especially in developing countries, prolonged rupture of the membranes and prolonged labour prior to surgery which increase the risk of endometritis. Imaging modalities such as ultrasonography (USG) and multidetector computed tomography (MDCT) are often used in the evaluation of suspected uncommon postcaesarean complications. It is often very difficult to differentiate between the normal and abnormal pelvic imaging findings in the immediate postoperative period. Various studies have been carried out to describe the normal and abnormal computed tomography (CT) findings of the female pelvis after caesarean section [4, 8-11]. Although CT has previously been found to be useless for uterine in uterine dehiscence, with the use of multiplanar reconstruction, especially in the sagittal plane, CT has proven to be good initial modality for assessing acute postoperative complications of caesarean section. MDCT can rapidly and comprehensively visualise surgically altered anatomy and triage the varied spectrum of possible complications [12]. Magnetic resonance imaging (MRI) may be required in doubtful cases of uterine dehiscence due to increased soft tissue resolution and better ability to visualize the serosa.

The objective of the research was to evaluate the imaging findings and the characteristic visual manifestations of uncommon acute complications of caesarean section, other than common complications such as postpartum haemorrhage, wound infection, etc.

\section{Materials and Methods}

This study was prospective and was carried out at the Department of Radiodiagnosis and Imaging, Government Medical College, Srinagar from June 2019 to February 2020 in collaboration with the Department of General Surgery and Gynaecology and Obstetrics of the Sher-i-Kashmir Institute of Medical Sciences (SKIMS). About 427 caesarean sections were performed from June 2019 to February 2020 at the Department of Gynaecology and Obstetrics, SKIMS. Out of 427 patients, 25 patients with suspected uncommon post-caesarean complications that could be detected by CT were included in the study.

\section{Surgical Technique}

After performing a Pfannenstiel incision, opening the fascia and separating the rectus muscles, assess into the peritoneum is gained. The Pfannenstiel incision is associated with less pain and loss of blood [1, 13, 14]. Thereafter, a space between the bladder and the uterus is created by incising the peritoneal reflection covering the uterus. This is followed by low transverse incision in the myometrium. Transverse incision is associated with lower rate of complications as compared to vertical incision. Vertical incision is associated with increased risk of rupture $[1,14]$. After delivering a newborn and placenta, suture is used to close the myometrium followed by fascial and skin closure.

\section{CT Protocol}

All patients with suspected complication in the immediate post-caesarean period were evaluated with contrast-enhanced MDCT. After baseline investigation, non-contrast images of the abdomen and pelvis were acquired followed by portal venous phase images at 45 to 60 seconds following the intravenous injection of $100 \mathrm{ml}$ of non-ionic low-osmolar contrast agent (omnipaque).

\section{Statistics}

Categorical variables were described in terms of frequency, percentage and mean.

\section{Results and Discussion}

The mean age of women undergoing caesarean section was 27.5 years. Sixteen patients had a history of one caesarean delivery, 7 patients had a history of two caesarean deliveries, two patients had no history of surgery. Out of 16 patients with a history of one caesarean delivery, one patient had history of myomectomy. Frequency and percentage of various complications in 25 patients were as follow (Table 1).

Table 1. Distribution of various complications in study patients.

\begin{tabular}{lcc}
\hline Complications & No. of patients & Percentage \\
\hline Bladder flap hematoma & 5 & $20 \%$ \\
Uterine dehiscence & 8 & $32 \%$ \\
Uterine rupture & 6 & $24 \%$ \\
Ureteral injury & 3 & $12 \%$ \\
Gossypiboma & 1 & $4 \%$ \\
Pelvic sepsis & 2 & $8 \%$ \\
\hline Total & 25 & $100 \%$ \\
\hline
\end{tabular}

The various imaging features of post-caesarean complications were as follows:

\section{Bladder Flap Hematoma}

Bladder flap hematomas rarely occur after lower segment caesarean sections. Bladder flap hematoma is a collection of blood between the urinary bladder and the lower uterine segment. On USG, hematoma is seen as a hyperechoic collection in the uterine-vesical space. On non-contrast CT with the mean attenuation of 40-70 $\mathrm{HU}$, hematoma is hyperdense. Hematomas less than $4 \mathrm{~cm}$ in size are common in up to $50 \%$ 


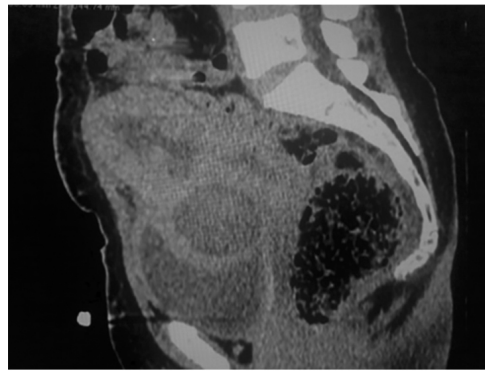

A

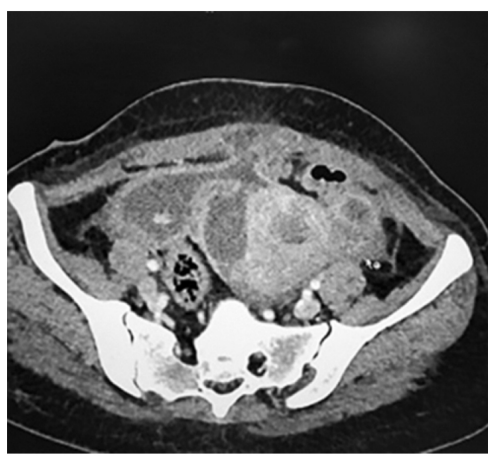

D

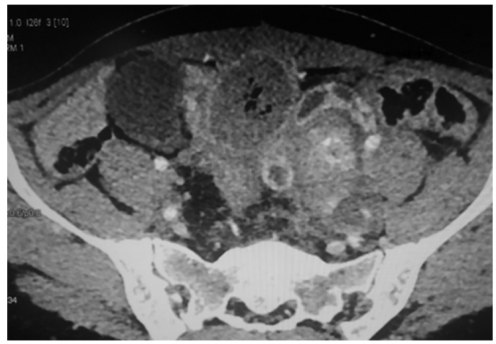

G

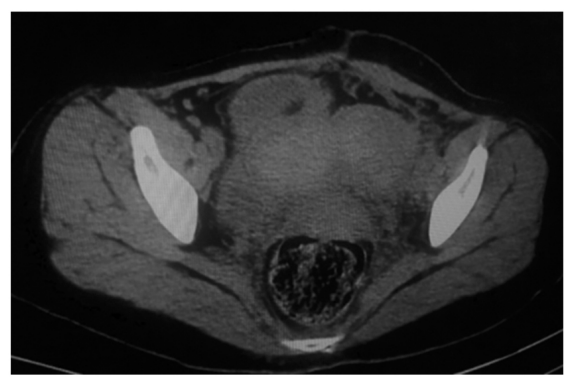

B

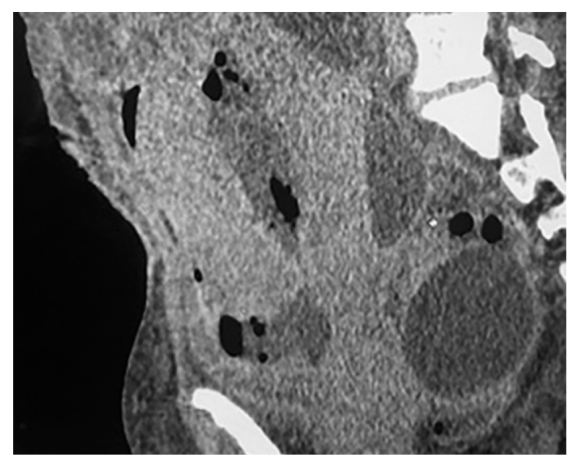

$\mathbf{E}$

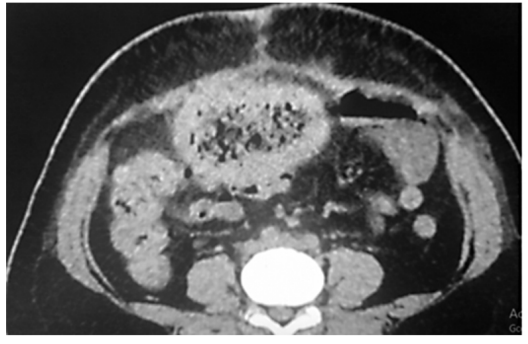

H

Figure 1. CT images showing acute post-caesarean section complications.

A - Sagittal contract CT showing a non-enhancing collection along the posterior bladder wall consistent with bladder flap hematoma in a post-caesarean patient.

B - Axial non-contract CT showing a hyperdense collection along the posterior bladder wall consistent with bladder flap hematoma in a post-caesarean patient.

C - Axial post-contrast CT images showing focal incomplete discontinuity of the myometrium consistent with uterine dehiscence in a post-caesarean patient.

D - Axial post-contrast CT images showing focal complete discontinuity of the myometrium with continuity between the endometrial cavity and parametrial collection consistent with uterine rupture in a post-caesarean patient.

E - Sagittal post-contrast CT images showing focal complete discontinuity of the myometrium with continuity of the endometrial cavity and parametrial collection consistent with uterine rupture in a post-caesarean patient.

F - Axial CT showing multiple gas accumulations in the pelvis consistent with postpartum abscesses.

G - Contrast-enhanced CT showing a well-defined area with air foci suggestive of an abscess with left-sided enhancing tubular structure suggestive of pyosalpinx.

H - Axial and sagittal non-contrast CT revealing the evidence of thick-walled, well-defined lesion with internal mottled air foci just beneath the caesarean scar and lost fat planes with gut suggestive of gossypiboma. 
of patients [15]. On CT, infected hematoma may present as a peripherally enhancing collection with internal air foci, with or without multiple internal septa, located in the expected region of simple bladder flap hematoma. In our study, 5 patients had bladder flap hematoma. Three out of 5 patients were treated by conservative management, one patient underwent percutaneous drainage after hematoma liquefaction, and one patient needed hematoma evacuation. Fig. 1A and 1B show a non-enhancing hyperdense collection between the uterus and bladder. One patient had hematoma measuring more than $4 \mathrm{~cm}$ and surgical evacuation was needed.

\section{Uterine Dehiscence/Uterine Rupture}

Uterine dehiscence occurs secondary to an incomplete rupture of the uterine wall involving the endometrium and myometrium with sparing the overlying serosa (Fig. 1C) [4]. It is often confused with normal post-caesarean appearance. There is also a fine difference between uterine dehiscence and rupture. Free fluid, bladder flap hematoma, pleural effusion, bowel dilatation, and liver abscess are described as associated signs [4]. Under appropriate clinical conditions, bladder flap hematoma $>5$, or large pelvic hematomas, raise suspicion for uterine dehiscence [4]. Uterine myometrial discontinuity gas is a normal post-operative finding. Visualization of uterine myometrial discontinuity gas on CT is not associated with febrile morbidity, caesarean characteristics, and trial of labor after caesarean results [16]. Focal disruption of the myometrium, the presence of gas containing track from the endometrium to the parametrium, and the addition of haemaperitoneum are imaging findings that typically provide the diagnosis of uterine rupture (Fig. 1D, 1E) [4, 11]. In our study, all cases of uterine rupture needed repeated laparotomy followed by repair, while most cases of uterine dehiscence were managed conservatively.

\section{Pelvic Sepsis}

Pelvic abscess occurs secondary to superimposed infection of a pelvic or bladder flap haematoma. On CT, an abscess is seen as a well-defined peripherally enhancing fluid attenuation lesion, with or without internal septations, containing varying amounts of internal echoes and gas (Fig. 1F, 1G). The presence of gas secondary to gas-producing organisms causes ringdown artefacts, leading to appearance of internal echoes with dirty posterior shadowing on ultrasound [4]. In our study, all patients with pelvic sepsis were treated by percutaneous drainage and appropriate antibiotic coverage.

\section{Ureteral Injury}

Ureteral injury is less frequent than bladder injury and goes unnoticed during caesarean section [17]. Rajasekar and Hall [17] reported that all bladder injuries were noted intraoperatively, whereas only 1 out of 4 ureteral injuries were noticed during the procedure as it co-existed with bladder injury. Other sources state that $70 \%$ of ureteral injuries remained undetected intraoperatively [18]. On CT imaging, ureteral injury can present with urinoma in the pelvis with contrast leakage on delayed phase imaging. In our study, all patients with ureteral injury were treated with percutaneous nephrostomy followed by ureteral stenting to allow for ureteral repair.

\section{Gossypiboma}

Retained surgical items (RSIs) are uncommon complication with the lowest incidence; however, they can lead to re-interventions in $70 \%$ of cases, with a morbidity and mortality being $80 \%$ and $35 \%$, respectively. Surgical sponges or gauze (gossypiboma or textiloma) are the most common RSIs, while cases of retained surgical instruments are rare [19]. Textiloma and gossypiboma are non-medical terms used to describe a mass of cotton matrix/sponge accidently left inside a body cavity during an operation. The term "gossypiboma" is derived from the Latin word gossypium, meaning cotton, and the Swahili word boma, meaning place of concealment [20, 21]. CT is the technique of choice for detecting gossypibomas. Fig. $1 \mathrm{H}$ presents $\mathrm{CT}$ finding of a low-density well-defined heterogeneous lesion with internal spongiform pattern of air foci and peripheral hyperdense wall that may show peripheral enhancement on contrast-enhanced imaging. In our study, single case of gossypiboma was treated with laparotomy followed by gossypiboma removal.

\section{Conclusions}

MDCT plays an important role in early detection and confirmation of multiple acute complications after caesarean delivery other than postpartum haemorrhage, wound infection, soakage, etc. and can also help in guiding the management of complications as well. Radiologists need to be familiar with normal postoperative findings such as small hematomas to avoid unnecessary repeated laparotomies and postoperative complications and to guide obstetricians in proper management of complications.

\section{Ethical Statement}

The study was conducted according to WHO Declaration of Helsinki - Ethical Principles for Medical Research Involving Human Subjects.

\section{Informed Consent}

Informed consent was obtained from each patient.

\section{Conflict of Interest}

The authors declare that no conflicts exist.

\section{Financial Disclosure}

The authors declared no financial support.

\section{References}

[1] Betrán AP, Merialdi M, Lauer JA, Bing-Shun W, Thomas J, Van Look P, et al. Rates of caesarean section: analysis 
of global, regional and national estimates. Paediatric and Perinatal Epidemiology [Internet]. 2007;21(2):98-113. Available from: 3016.2007.00786.x

[2] Dresang LT, Leeman L. Cesarean delivery. Primary Care: Clinics in Office Practice [Internet]. 2012;39(1):145-165. Available from: https://doi.org/10.1016/j.pop.2011.11.007

[3] Rebelo F, da Rocha CMM, Cortes TR, Dutra CL, Kac G. High cesarean prevalence in a national populationbased study in Brazil: the role of private practice. Acta Obstetricia et Gynecologica Scandinavica [Internet]. 2010;89(7):903-908. Available from: https://doi.org/10.3109/00016349.2010.484044

[4] Rodgers SK, Kirby CL, Smith RJ, Horrow MM. Imaging after cesarean delivery: acute and chronic complications. RadioGraphics [Internet]. 2012;32(6):1693-1712. Available from: https://doi.org/10.1148/rg.326125516

[5] Gonzalo-Carballes M, Ríos-Vives MÁ, Fierro EC, Azogue XG, Herrero SG, Rodríguez AE, et al. A pictorial review of postpartum complications. RadioGraphics [Internet]. 2020;40(7):2117-2141. Available from: https://doi.org/10.1148/rg.2020200031

[6] Gui B, Danza FM, Valentini AL, Laino ME, Caruso A, Carducci B, et al. Multidetector CT appearance of the pelvis after cesarean delivery: normal and abnormal acute findings. Diagnostic and Interventional Radiology [Internet]. 2016;22(6):534-541. Available from: https://doi.org/10.5152/dir.2016.15593

[7] Deng M-F, Zhang X-D, Zhang Q-F, Liu J. Uterine rupture in patients with a history of multiple curettages: two case reports. World Journal of Clinical Cases [Internet]. 2020;8(24):6322-6329. Available from: https://doi.org/10.12998/wjcc.v8.i24.6322

[8] Twickler DM, Setiawan AT, Harrell RS, Brown CE. CT appearance of the pelvis after cesarean section. American Journal of Roentgenology [Internet]. 1991;156(3):523-526. Available from: https://doi.org/10.2214/ajr.156.3.1899749

[9] Hiller N, Schor-Bardach R, Gileles-Hillel A, Stroumsa D, Simanovsky N. CT appearance of the pelvis after Cesarean delivery - what is considered normal? Clinical Imaging [Internet]. 2013;37(3):514-519. Available from: https://doi.org/10.1016/j.clinimag.2012.09.022

[10] Kamaya A, Ro K, Benedetti NJ, Chang PL, Desser TS. Imaging and diagnosis of postpartum complications. Ultrasound Quarterly [Internet]. 2009;25(3):151-162. Available from: https://doi.org/10.1097/RUQ.0b013e3181b5451e
[11] Sierra A, Burrel M, Sebastia C, Radosevic A, Barrufet $\mathrm{M}$, Albela $\mathrm{S}$, et al. Utility of multidetector CT in severe postpartum hemorrhage. RadioGraphics [Internet]. 2012;32(5):1463-1481. Available from: https://doi.org/10.1148/rg.325115113

[12] Tonolini M. Multidetector CT of expected findings and complications after hysterectomy. Insights into Imaging [Internet]. 2018;9(3):369-383. Available from: https://doi.org/10.1007/s13244-018-0610-9

[13] Hofmeyr JG, Novikova N, Mathai M, Shah A. Techniques for cesarean section. American Journal of Obstetrics and Gynecology [Internet]. 2009;201(5):431-444. Available from: https://doi.org/10.1016/j.ajog.2009.03.018

[14] Bates GW, Shomento S. Adhesion prevention in patients with multiple cesarean deliveries. American Journal of Obstetrics and Gynecology [Internet]. 2011;205(6):S19-S24. Available from: https://doi.org/10.1016/j.ajog.2011.09.030

[15] DiFlorio-Alexander R, Harris RD. Postpartum complications. In: Fielding JR, Brown DL, Thurmond AS, editors. Gynecologic Imaging. Philadelphia, Pa: Elsevier; c2011. 381-398. Available from: https://doi.org/10.1016/B978$1-4377-1575-0.10025-8$

[16] Bogot NR, Rottenstreich M, Nabulsi RM, Turner Y, Farkash R, Samueloff A, et al. The radiological myometrial appearance after cesarean section: is it associated with postpartum complications and subsequent delivery outcome? Abdominal Radiology [Internet]. 2017;43(8):2150-2155. Available from: https://doi.org/10.1007/s00261-017-1408-9

[17] Rajasekar D, Hall M. Urinary tract injuries during obstetric intervention. BJOG: An International Journal of Obstetrics and Gynaecology [Internet]. 1997;104(6):731-734. Available from: https://doi.org/10.1111/j.1471-0528.1997.tb11986.x

[18] Mann WJ, Arato M, Patsner B, Stone ML. Ureteral injuries in an obstetrics and gynaecology training program: etiology and management. Obstet Gynecol. 1988;72(1):82-85.

[19] Gavrić Lovrec V, Cokan A, Lukman L, Arko D, Takač I. Retained surgical needle and gauze after cesarean section and adnexectomy: a case report and literature review. Journal of International Medical Research [Internet]. 2018;46(11):4775-4780. Available from: https://doi.org/10.1177/0300060518788247

[20] Jain M, Jain R, Sawhney S. Gossypiboma: ultrasoundguided removal. Journal of Clinical Ultrasound [Internet]. 1995;23(5):321-323. Available from: https://doi.org/10.1002/jcu.1870230509 
[21] Rasoaherinomenjanahary F, Ratsimarisolo N, Ramamonjiharisoa AG, Rakotondrainibe A, Ranoharison DH, Andriantsoa MJ, et al. Abdominal textiloma mimicking as left colic tumour: a postoperative complication still common in low-income countries. EMJ Radiology [Internet]. 2020;1(1):72-78. Available from: https://doi.org/10.33590/emjradiol/19-00208
Received: 2020-12-25

Revised: 2021-04-19

Accepted: 2021-05-05 